\title{
Zielvorgaben sind Budgets - zum Nachteil der Patienten
}

\author{
Jürg Schlup \\ Dr. med., Präsident der FMH
}



Wer sich gerne auf Basis von Fakten eine Meinung bildet, konnte im Februar eine erfreuliche Meldung zu unserem Gesundheitswesen vernehmen: Erneut lag dieses in einem internationalen Ranking an der Spitze, dank seines hohen Standards "in allen Bereichen" und des "exzellenten Zugangs» $\mathrm{zu}$ "sehr guter medizinischer Qualität» [1, S. 28]. Dies dürften die Gründe sein, warum aktuell 87\% der Bevölkerung das Gesundheitswesen positiv beurteilen - so viele wie noch nie [2]. Eine Herausforderung bleibt der Preis dieses Gesundheitswesens. Auch hier ist zwar die Situation deutlich besser als oft behauptet: 2017 stiegen die Kosten der Krankenversicherung nur noch um 1,7\% [3]; 2018 stagnierten sie sogar [4]. Der Anteil der Versicherten, die die Prämien als «dauerhaftes Problem» bezeichnen, sank auf 5\% - einen historischen Tiefstand [2]. Zudem liegen Vorschläge vor, wie auch langfristig Ressourcen möglichst effizient eingesetzt und die Prämien so gering wie möglich gehalten werden können ohne die Versorgungsqualität und die Wahlfreiheit der Patienten einzuschränken [5].

In der Regel sind Sparbemühungen jedoch mit Zielkonflikten verbunden, so auch im Gesundheitswesen: Alle wünschen sich möglichst geringe Gesundheitskosten und niedrige Prämien - die meisten lehnen aber gleichzeitig Reduktionen des Leistungsangebots oder Einschränkungen des Zugangs ab [6]. Dieses eigentlich klassische Dilemma haben einige politische Akteure in ihrem Sinne neu interpretiert und sogar für den Wahl-

\section{Hinter der unverdächtigen Zielvorgabe versteckt sich ein Budgetdeckel: Betroffene Patienten werden aus allen Wolken fallen.}

kampf nutzbar gemacht: Sie versprechen einfach das Füfi und das Weggli. Spürbare Kostendämpfung ohne wahrnehmbare Einschränkungen, also gleiche Leistung für weniger Geld, lautet ihr Versprechen.

Ihre Aussagen klingen dabei so, dass spontan jeder zustimmen möchte: Die Gesundheitskosten dürften nicht stärker steigen als die Löhne (CVP); man benötige «Zielvorgaben für die Ausgabenentwicklung» (EDI-Experten); man müsse «ungerechtfertigte Erhöhungen der Mengen und der Kosten» (Vorschlag Art 47c, KVG) [7] verhindern oder ein "zu hohes Kostenwachstum» durch Eingriffe in Tarifverträge bekämpfen (Motion 18.3305). All dies klingt attraktiv, denn niemand wehrt sich gegen Zielvorgaben - oder verteidigt überzogene Kosten. Tatsache ist aber: Bei all diesen Forderungen werden der Gesundheitsversorgung Budgets auferlegt, um Geld zu sparen - und welche Kosten «ungerechtfertigt» [7] sind, definiert die Politik, nicht der Patient.

Klar zu sagen, was bei wem eingespart werden soll, würde Wähler abschrecken.

Wie das Wunder des völlig schmerzfreien Sparens dann praktisch vollbracht werden soll, bleibt selbstverständlich offen: $\mathrm{Zu}$ sagen, was bei wem eingespart werden soll, würde die Wähler abschrecken. Lieber verweist man allgemein auf ein - komplett unbelegtes Sparpotential von 20\% im Gesundheitswesen. Die CVP schiebt den schwarzen Peter für konkrete Lösungen Bund und Kantonen zu, die nicht näher spezifizierte «Massnahmen» ergreifen sollen. Die anderen oben zitierten Vorschläge möchten hingegen uns Ärzte über den Tarif zwingen, bei politisch nicht gewollten Kosten zu rationieren.

Unbequeme Tatsache ist, dass es Kostendämpfung ohne Einschränkungen in der Regel nicht gibt. Verständlich darum der Wunsch, den eine Hausärztin aus dem Kanton Glarus im Interview auf Seite 366 [8] äussert: Patienten sollten nicht nur die «Prämien anschauen, sondern sich auch über die geplanten Neuerungen im Gesundheitswesen informieren». Patienten, die bereits heute angesichts der limitierten Konsultationszeiten "aus allen Wolken" fallen, würden in einer budgetierten Versorgung noch mehr böse Überraschungen erleben.

«Dass wir in der Schweiz noch kein Globalbudget für Patienten haben» schätzt die aus Deutschland eingewanderte Kollegin als grossen Vorteil - und die internationalen Rankings sowie die hohe Zufriedenheit der Bevölkerung geben ihr Recht. Wir dürfen unsere TopPatientenversorgung nicht zugunsten leerer Versprechungen einschränken. 